\title{
Optimal Aperture Distribution for Near-field Detection of Foreign Objects in Lossy Media
}

\author{
A. Razavi*
}

R. Maaskant*

\begin{abstract}
We determine the optimal source aperture field distribution to maximize the power transfer through a lossy medium from the transmitter aperture to a receiver aperture located in the nearfield region. The optimal distribution is determined by applying array signal processing techniques to aperture field modes. By designing the system to be sensitive to the presence of foreign objects in a homogenous matter, the synthesized optimal aperture distribution increases the detection probability of foreign objects in various near-field applications, such as those that can detect potentially hazardous contaminations in food.
\end{abstract}

\section{INTRODUCTION}

Microwave systems are increasingly used in many detection and sensing applications. Hence, the need for antennas that are specifically designed for nearfield applications is brought upon. When it comes to detecting foreign objects in lossy homogenous matters, different approaches can be chosen to determine the optimal antenna characteristics. The $3 \mathrm{~dB}$ near-field beam radius is introduced in [1] and utilized to find the optimal aperture size for uniform field apertures for the detection of foreign objects in lossy media [2]. Another approach, which is chosen in the current work, is to maximize the power coupling between a pair of antennas acting as transmitter and receiver, as this will enhance the system sensitivity in detecting the presence of a foreign object.

Kay [3] investigated the near-field gain of aperture antennas in an attempt to maximize it. However, the analytical approach falls short when determining the exact optimal aperture field distribution due to its computational complexity. Using a different approach, Borgiotti [4] utilized the reaction integrals in order to formulate and maximize the power transfer between two planar apertures in the near-field. However, the field distribution in that approach can only be solved analytically for the particular case of rectangular apertures. Recently, Sanghoek et al. [5] used a two-port network model to optimize the coupling efficiency between an infinitely large sheet of magnetic currents and a combination of electric and magnetic dipoles through a

\footnotetext{
*Department of Signals and Systems, Chalmers University of Technology, Gothenburg, Sweden, e-mail: aidin.razavi@chalmers.se, rob.maaskant@chalmers.se, jian.yang@chalmers.se, mats.viberg@chalmers.se
}

planar multilayered medium.

We propose an array signal processing technique applied to aperture field modes in order to find the transmitter aperture field distribution that maximizes the power coupling between the two antennas in the near-field. The numerical method is generic and allows us to analyze arbitrarily shaped antenna aperture geometries [6]. In this paper, it will be demonstrated that the system sensitivity due to the presence of a foreign object is highest when the optimal aperture field distribution is used.

\section{PROBLEM DESCRIPTION AND OP- TIMIZATION PROCEDURE}

Consider the RF detection system in Fig. 1, comprising of a transmitting antenna array and a smaller receiving antenna that are separated by a lossy medium. The intrinsic impedance of the medium is $\eta$ and represents a homogeneous flow of lossy matter through the sensing system, or a homogeneous solid object, which may, at times, be contaminated by a foreign object. The objective is to determine an optimal antenna system to detect the presence of the foreign object with high probability. The proposed approach is to maximize the coupling between the transmitting and receiving antennas in the absence of the foreign object, thereby rendering the system very sensitive to the situation when the foreign object is present, so that it can be detected with high sensitivity by measuring the change in the antenna coupling coefficient.

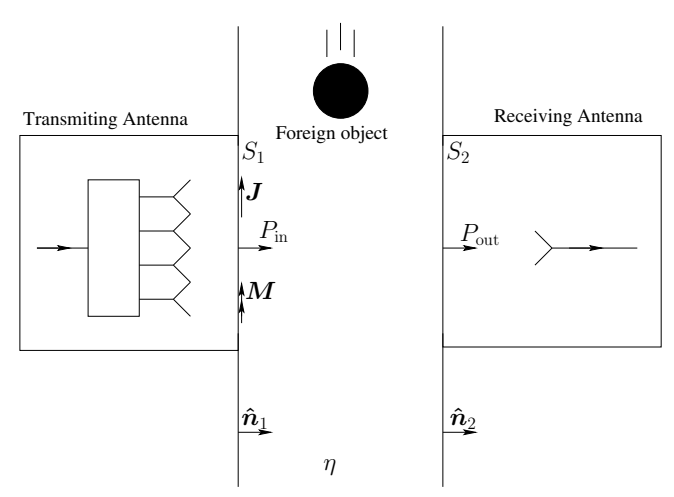

Figure 1: Antenna system for the detection of foreign objects in a lossy medium. 
The goal of determining the optimal aperture is to find the source current distribution on the transmitting aperture that results in the maximum received power. This will provide us with the best possible coupling (upper bound) between two antennas in the near-field in the absence of an object. The focus of the present paper is on the theoretical analysis, while the realization of the optimal antenna will be left for future report.

By expanding the unknown transmitter aperture distribution, which is given as a combination of electric $(\boldsymbol{J})$ and magnetic $(\boldsymbol{M})$ currents, in terms of $2 N$ basis functions with unknown weights as

$$
\boldsymbol{J}=\sum_{n=1}^{N} j_{n} \boldsymbol{f}_{n}(\boldsymbol{r}), \quad \text { and } \quad \boldsymbol{M}=\sum_{n=1}^{N} m_{n} \boldsymbol{g}_{n}(\boldsymbol{r})
$$

it can be shown that the power transfer ratio between the two apertures can be written in matrix form as a ratio of quadratic forms, i.e., (see also [6])

$$
P_{\mathrm{tr}}=\frac{P_{\mathrm{out}}}{P_{\mathrm{in}}}=\frac{\mathbf{w}^{H} \mathbf{P}_{\mathrm{out}} \mathbf{w}}{\mathbf{w}^{H} \mathbf{P}_{\mathrm{in}} \mathbf{w}}
$$

where $\mathbf{P}_{\text {in }}$ and $\mathbf{P}_{\text {out }}$ are Hermitian system matrices relating the input and output powers, respectively, to the transmitting aperture field distribution and $\mathbf{w}=\left[j_{1}, j_{2}, \ldots, j_{N}, m_{1}, m_{2}, \ldots, m_{N}\right]^{T}$ is the vector containing the unknown weights of the different field modes. By knowing w, the transmitting aperture field distribution can be calculated. The objective is to determine $\mathbf{w}$ so as to maximize the power transfer ratio, which leads to solving the generalized eigenvalue equation [7]

$$
\mathbf{P}_{\text {out }} \mathbf{w}=P_{\text {tr }} \mathbf{P}_{\text {in }} \mathbf{w} .
$$

Hence the maximization of $P_{\mathrm{tr}}$ is equivalent to finding the largest eigenvalue in (3). Once the optimal $\mathbf{w}$ is found, the aperture field distribution is computed through the aperture field modes.

\section{RESULTS}

Henceforth, fat $\left(\epsilon_{r}=4.6, \sigma=0.02 \mathrm{~S} / \mathrm{m}\right.$ at $\left.1 \mathrm{GHz}\right)$ is used as the lossy matter separating the apertures. Also, since the focus in this work is on the design of the transmitter antenna, we assume the receiver as an $0.5 \lambda \times 0.5 \lambda$ ideally conjugately matched antenna aperture harvesting all the power passing through it for simplicity. The transmitter is assumed to be a $4 \lambda \times 4 \lambda$ square aperture and the spacing between the two antennas is chosen to be $4 \lambda$. Furthermore, the transmitter antenna is assumed to be $x$-polarized. Fig. 2 shows the normalized distribution of radiated power on the transmitter aperture for the eigenmodes corresponding to the five

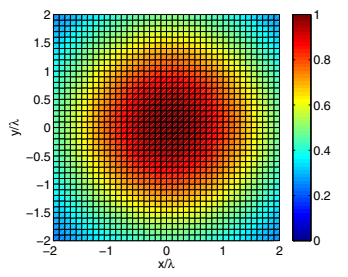

mode 1 (optimal)

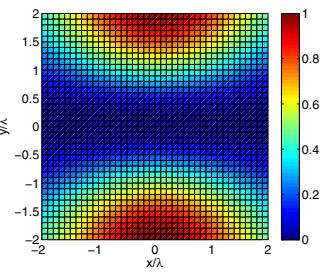

mode 2



mode 4



mode 3

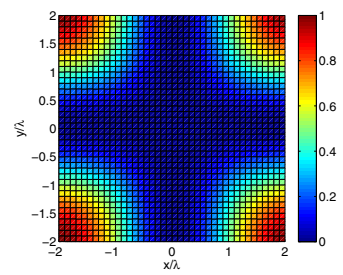

mode 5
Figure 2: Normalized radiated power corresponding to the five largest positive eigenvalues of (3).



Figure 3: Simulated $P_{\mathrm{tr}}$ of first five modes. The $P_{\mathrm{tr}}$ for the uniform distribution is included as a horizontal line.

largest positive eigenvalues of (3). These aperture field distributions are modeled in CST and the corresponding $P_{\mathrm{tr}}$ is plotted in Fig. 3. The uniform field distribution which provides the highest directivity in the far-field and which is relatively easy to realize is also included in this figure as a horizontal line for the sake of comparison. Fig. 3 shows more than $14 \mathrm{~dB}$ advantage in $P_{\mathrm{tr}}$ for the optimal distribution over the second best eigenmode and more than $6 \mathrm{~dB}$ over the uniform field distribution, the latter of which can be expanded as a weighted sum of multiple eigenmodes.

Earlier, we have proposed the hypothesis that 


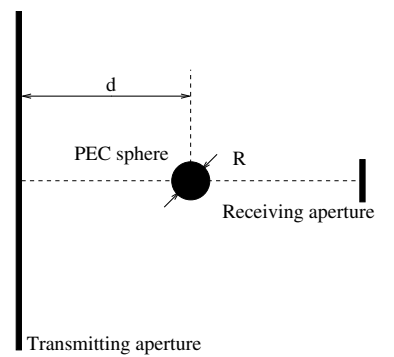

Figure 4: Introduction of a PEC sphere of radius $R$ at distance $d$ from transmitter aperture.

maximizing the power coupling between the two antennas in a homogenous medium helps increasing the system sensitivity to detecting the presence of a foreign object. In order to verify this, we introduce a PEC sphere of radius $R$ into the space between the two antennas as in Fig. 4 and compute the power transfer ratio in the presence of this object. Next, we define the difference in power transfer ratio $\Delta P_{\mathrm{tr}}$ for each mode as

$$
\Delta P_{\mathrm{tr}}=\left|P_{\mathrm{tr}}^{\mathrm{sphere}}-P_{\mathrm{tr}}^{\text {free }}\right|=\frac{\left|P_{\text {out }}^{\text {sphere }}-P_{\mathrm{out}}^{\text {free }}\right|}{P_{\text {in }}}
$$

where $P_{\mathrm{tr}}^{\text {sphere }}$ and $P_{\mathrm{tr}}^{\text {free }}$ are the power transfer ratio for each mode in the presence and absence of the PEC sphere, respectively, and $P_{\text {in }}=P_{\text {in }}^{\text {sphere }}=$ $P_{\text {in }}^{\text {free }}$ is the input power due to electric and magnetic current sources. $\Delta P_{\mathrm{tr}}$ translates to the change in measured $S_{21}$ when the two antennas are connected to a network analyzer in a detection system, and it is obvious that a larger $\Delta P_{\mathrm{tr}}$ will increase the possibility of the detection of a foreign object in the medium. $\Delta P_{\mathrm{tr}}$ for both the first five modes and the uniform field distribution, are plotted in Fig. 5 for $R=0.25 \lambda$ and $d=2 \lambda$. It is observed that $\Delta P_{\mathrm{tr}}$ is highest when the optimal transmitter aperture field distribution is used and it occurs at a larger $P_{\mathrm{tr}}$ value, which further implies better SNR in the measurements. As any aperture field distribution can be expanded as a weighted sum of all aperture modes, including those with even lower values of $P_{\mathrm{tr}}$, the $\Delta P_{\mathrm{tr}}$ of any transmitter field distribution is likely to be smaller than that of the optimal aperture. It is worth mentioning that, although the modes 2 and 3 seem to have similar aperture field distributions as well as an equal $P_{\mathrm{tr}}$, the difference in $\Delta P_{\mathrm{tr}}$ of these two modes is due to the $x$-polarization of the fields. As the radiated field by mode 2 is mostly tangential to the PEC surface, making it a soft surface [8], while for mode 3 it is mostly normal to the PEC surface, forming a hard surface.

In order to gain a better insight in the radiated

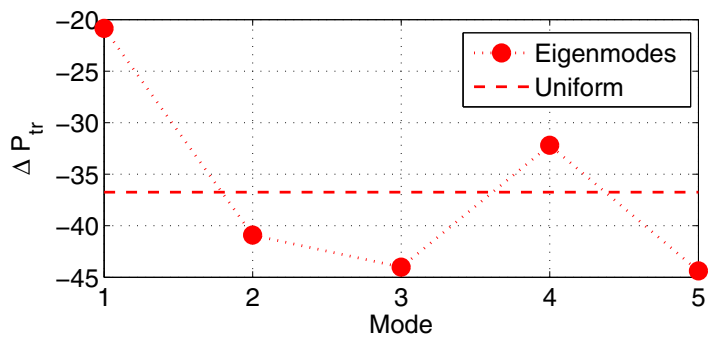

Figure 5: $\Delta P_{\mathrm{tr}}$ for different aperture field distributions. Uniform distribution is included as a horizontal line.
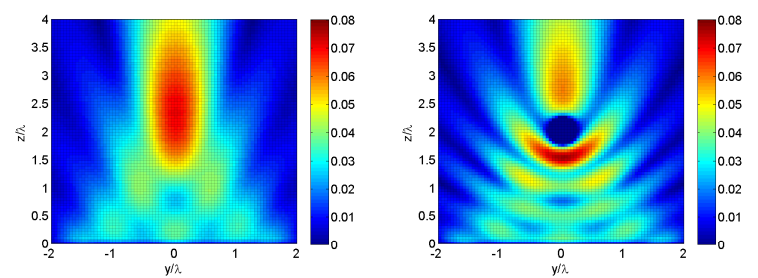

(a) mode 1
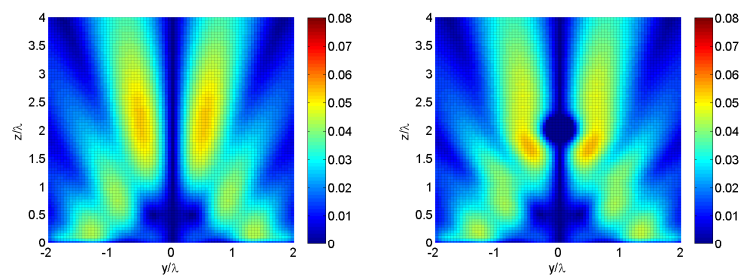

(b) mode 3


(c) uniform

Figure 6: E-field intensity in the $x=0$ plane radiated by the optimal distribution, mode 3 and the uniform field aperture in the absence (left column) and presence (right column) of the PEC sphere.

mode fields, the intensity of the E-field in the $x=0$ plane, in the absence and presence of the PEC sphere, are plotted in Fig. 6 for the optimal distribution, mode 3 , and the uniform distribution as two representative distributions. Clearly, the radiated field and the field on the receiver aperture in the case of optimal aperture are distorted more by the presence of a foreign PEC object as compared to the other aperture field distributions. 


\section{CONCLUSIONS}

Array signal processing techniques have been applied to aperture field modes in order to synthesize the optimal aperture field distribution that maximizes the power coupling between a transmitter and a smaller receiver antenna aperture located in the near-field of one another and in a lossy medium. It has been shown that by maximizing the power coupling between both antennas, the detection possibility of foreign objects in homogenous lossy media is increased.

\section{References}

[1] A. Razavi, J. Yang, and T. McKelvey, "Optimal Aperture Distribution of Near-Field Antennas for Maximum Signal Penetration", 7th European Conference on Antennas and Propagation, EUCAP 2013, Gothenburg, Sweden, April 2013.

[2] A. Razavi, and J. Yang, "Optimal Size of Uniform Aperture for Near-field Penetration Through Lossy Medium", Microwave and $\mathrm{Op}$ tical Technology Letters, In press.
[3] A. F. Kay, "Near-field Gain of Aperture Antennas", IRE Trans. Antennas Propag., vol. 8, no. 6, pp. 586-593, 1960.

[4] G. V. Borgiotti, "Maximum Power Transfer Between Two Planar Apertures in Fresnel Zone", IEEE Trans. Antennas Propag., vol. 14, no. 2, pp. 158-163, 1966.

[5] K. Sanghoek, J.S. Ho, and A.S.Y. Poon, "Wireless Power Transfer to Miniature Implants: Transmitter Optimization", IEEE Trans. Antennas Propag., vol. 60, no. 10, pp. 4838-4845, 2012.

[6] A. Razavi, R. Maaskant, J. Yang, and M. Viberg, "Maximum Aperture Power Transmission in Lossy Homogeneous Matters", submitted to IEEE Antennas Wireless Propag. Lett.

[7] R. F. Harrington, Field Computation by Moment Methods. New York: The Macmillan Company, 1968.

[8] P.-S. Kildal, "Artificially Soft and Hard Surfaces in Electromagnetics", IEEE Trans. Antennas Propag., vol. 38, no. 10, pp. 1537-1544, 1990. 